The efiect of oestrogens and progestogens on serum protein levels

C. H. W. HORNE, ANNETTE C. MALLINSON, AND R. B. GOUDIE (University Department of Pathology, Western Infirmary, Glasgow)

In a previous study the levels of four serum proteins, assayed by a radial immunodiffusion technique, were measured in nine healthy women before and after taking combined oestrogen/progestogen oral contraceptives. Significant increases in $\alpha_{2}$ macroglobulin, transferrin, and IgG were found but albumin was unchanged. The purpose of the present experiment was to determine whether the oestrogen or progestogen component was responsible for these changes.

Two groups of 14 healthy females were given either $50 \mathrm{mg}$ oestrogen (mestranol) or $1 \mathrm{mg}$ progestogen (ethynodiol diacetate) daily for three weeks. Protein levels were determined, at weekly intervals, before, during, and after therapy. Significant increases in $\alpha_{2}$ macroglobulin and transferrin were found in those subjects taking mestranol. Neither of the two groups showed significant changes in IgG or albumin.

Protein levels in 14 healthy females were measured at three- to fourday intervals over one menstrual cycle. No convincing evidence of cyclical variation due to endogenous production of hormones was found but there was some indication that transferrin perhaps showed cyclical variation.

\section{The pepsins of patients with peptic ulcer}

W. H. TAYLOR (Department of Chemical Pathology, The United Liverpool Hospitals, Ashton Street, Liverpool 3)

Agar gel electrophoresis at $p \mathrm{H} 5.0$ of histamine-stimulated normal gastric juice reveals eight proteolytic zones, not all of which are present in every subject (see Table). Zones 1, 2, 3, 3a, and 5 are caused by different individual pepsins, of which 3 and 5 are the principal pepsins. Zones 4 and 6 arise from a pepsin inhibitor complex and from residual zymogen respectively. Zone 7 is not inactivated at alkaline $p H$ and is better called a gastric proteinase than a pepsin.
Pepsin 1 occurs with significantly increased frequency in patients with peptic ulcer (Table). It also occurs in several such patients in greatly increased amounts. Patients with gastric ulcer exhibit these effects to a greater extent than patients with duodenal ulcer.

There is no association of hyperchlorhydria with increasing amounts of pepsin 1 ; in duodenal ulcer the data suggest the reverse, but are not conclusive. Nor is there any association between the presence of pepsin 1 and the possession of blood group $\mathbf{O}$; in both sorts of ulcer the data again suggest the reverse.

A new aetiological factor in peptic ulcer has thus been revealed, which is operating more frequently in gastric than in duodenal ulceration, and independently of other aetiological factors, such as hyperchlorhydria and the possession of blood group $\mathbf{O}$.

Infection due to Klebsiella aerogenes in a neurosurgical unit

\section{PRICE AND J. D. SLEIGH (Glasgow)}

Sixteen of the 75 beds in the West of Scotland neurosurgical unit form an intensive care ward and it was here that infection was most serious. The causal organism was shown biochemically to be Klebsiellaa aerogenes and 114 of 217 isolates from different sources had the same antibiotic sensitivity pattern. However, 33 different patterns were recognized, colistin and gentamicin being the only antibiotics to which all strains were sensitive.

Infection with $K$. aerogenes, first recognized late in 1967 , became serious in the summer of 1968 when, in the intensive care ward, one patient in four had a chest infection and one patient in eight a urinary infection due to this organism. During the next 10 months patients with these infections received conventional antibiotic treatment based on the sensitivity tests in vitro but less than $10 \%$ of them showed any improvement. Twelve patients died from Klebsiella infections, eight of them from meningitis.

Infections were then treated with colistin in six times the maximum recommended dose and, although individual patients benefited, Klebsiella infection was not

\begin{tabular}{lllllllll}
\hline & $\begin{array}{l}\text { No. of } \\
\text { Patients }\end{array}$ & \multicolumn{3}{l}{ Pepsin Zones } & & & \\
\cline { 2 - 8 } & & 7 & 6 & 5 & 4 & 3 & 2 & 1 \\
\hline Normal subjects & 59 & 19 & 3 & 100 & 58 & 100 & 29 & 47 \\
Patients with gastric ulcer & 37 & 21 & 3 & 100 & 54 & 97 & 46 & 78 \\
Patients with duodenal ulcer & 31 & 0 & 0 & 100 & 48 & 100 & 32 & 68 \\
\hline
\end{tabular}

Table Frequency of occurrence $(\%)$ 